\title{
TOPOLOGICAL $K$-THEORY OF THE INTEGERS AT THE PRIME 2
}

\author{
LUKE HODGKIN
}

\author{
(communicated by James Stasheff)
}

\begin{abstract}
Recent results of Voevodsky and others have effectively led to the proof of the Lichtenbaum-Quillen conjectures at the prime 2, and consequently made it possible to determine the 2-homotopy type of the $K$-theory spectra for various number rings. The basic case is that of $B G L(\mathbb{Z})$; in this note we use these results to determine the 2-local (topological) $K$-theory of the space $B G L(\mathbb{Z})$, which can be described as a completed tensor product of two quite simple components; one corresponds to a real 'image of $J$ ' space, the other to $B B S O$.
\end{abstract}

\section{Introduction}

As a result of Voevodsky's solution of the Milnor conjecture [V] and related work by Bloch, Lichtenbaum, Voevodsky and Suslin [B-L], [S-V], Weibel in [W] calculated the algebraic $K$ theory of the integers $\pi_{i}\left(B G L(\mathbb{Z})^{+}\right)$at the prime 2 in terms which essentially confirmed the appropriate version of the Lichtenbaum-Quillen conjectures [L,D-F]. (Much stronger and more general versions of the prime-2 conjectures have since been proved, see in particular $[\mathrm{RWK}],[\mathrm{R}$ W].) This result, since it expresses the space $B G L(\mathbb{Z})^{+}$in terms of rather well-known spaces, makes it relatively easy to deduce other invariants. Arlettaz et al. in [A-M-N-Y] have done this for the mod 2 cohomology; in this paper, I shall do the same for the (topological) 2-local $K$-theory; the result, which is, perhaps predictably, quite different from that for cohomology is stated in theorem 4.1 and corollary 5.1 below. The use of 2-local, rather than the more usual 2-complete theory requires a little more work, but perhaps can be considered as giving a more interesting result.

While Weibel's results are more general in character, and could lead to similar calculations for various other rings e.g. $\mathbb{Z}[\sqrt{-1}]$, I shall here confine my attention to the integers, partly because of their 'historical' interest, and partly because of the link with the stable mapping class group $B \Gamma=\lim B \Gamma_{n}$ via the composite $B \Gamma \rightarrow B S p(\mathbb{Z}) \rightarrow B G L(\mathbb{Z})$, which arises from the action of surface homeomorphisms on $H_{1}$.

Without attempting a complete survey of recent related work, I should draw attention to the most important:

(i) The corresponding decomposition of spectra for the $p$-adics $B G L\left(\mathbb{Z}_{\hat{p}}\right)$ has been known for some time - for arbitrary $p$ - through work of Bökstedt, Madsen and Rognes [B-M], [R2]. (Here the Milnor conjecture is not needed.)

(ii) Dwyer and Mitchell, in a sequence of papers, [D-M], [Mi1], [Mi2], have attacked precisely the problem of finding the $K$-theory of the spectrum associated with $B G L(R)_{p} \hat{)}$ when $R$ is a ring of algebraic integers, which they have (essentially) solved in terms of the 'Iwasawa module' $M_{\infty}$ of $R$. The remaining dificulties are those associated with the structure of $M_{\infty}$, and are not trivial.

Received 26 May 2000, revised 19 October 2000; published on 13 November 2000.

2000 Mathematics Subject Classification: 19D99, 55N15, 55P15.

Key words and phrases: K-theory, general linear group of integers, Rothenberg-Steenrod spectral sequence, Bousfield localization.

(C) 2000, Luke Hodgkin. Permission to copy for private use granted. 
(iii) In an important special case, Østvær [Ø] has found the homotopy type of $B G L(R)_{2} \hat{}$ when $R$ is '2-regular and non-exceptional'. Examples include cyclotomic rings $R=\mathbb{Z}\left[\zeta_{2^{r}}\right], r \geqslant$ 2, (in particular the Gaussian integers); but no rings of integers in totally real fields. The homotopy type is very simple, a product of a $K\left(\mathbb{F}_{p}\right)$ (finite field) and copies of $U, S U$.

Having said this, the result presented here is perhaps principally of interest in the way that it displays the intertwining of the factors which go to make up $B G L(\mathbb{Z})$. The justification which Dwyer and Mitchell give for studying the topological $K$-theory of algebraic spectra (that it sheds light on the $K$-localization of the spectra, and hence can provide evidence about the Lichtenbaum-Quillen conjectures) is not really at issue at the prime 2 where the conjectures are solved; however, the structure has independent interest.

We begin by describing the space which we shall study. Following [Bö], but working in the category of 2-local spaces, we define the 'étale $K$-space' $J K(\mathbb{Z})$ as the (2-local) homotopy fibre of the composite map:

$$
c\left(\psi^{3}-1\right): B O \stackrel{\psi^{3}-1}{\longrightarrow} B \operatorname{Spin} \stackrel{c}{\longrightarrow} B S U
$$

(Here $\psi^{3}$ is the Adams operation and $c$ denotes complexification.) This space can be realized through a number of other fibrations, of which we shall note particularly (cf [R2], (2.3))

$$
J R_{2} \rightarrow J K(\mathbb{Z}) \rightarrow B B S O
$$

where $J R_{2}$ is the real image of $J$ space at 2, defined as the fibre of $\psi^{3}-1: B O \rightarrow B$ Spin, localized at 2. (See e.g. [Ma].) The 2-completion of $J K(\mathbb{Z})$ is equivalent to the space which is named $K^{e ́ t}(\mathbb{Z})$ in $[\mathrm{D}-\mathrm{F}]$ and elsewhere. Bökstedt defined a map on 2-completions from $\left(\left(B G L(\mathbb{Z})^{+}\right)_{2}\right.$ to $J K(\mathbb{Z})_{2} ;$ it is a consequence of Voevodsky's theorem and subsequent work that this is a homotopy equivalence. However, it is not obvious that this map is an equivalence - even that it exists - in the localized sense. [I am grateful to the referee for pointing this fact out.] I shall therefore, in sections 2 -4, find the $K$-theory of $J K(\mathbb{Z})$ with coefficients in $\mathbb{Z}_{2}$ and $\mathbb{Z}_{2}$, by a simple application of the Rothenberg-Steenrod spectral sequence. Having done that, in $\S 5 \mathrm{I}$ shall deduce the corresponding results for $B G L(\mathbb{Z})^{+}$; the completed case is easy, by the above remarks, but the local case requires a special investigation, using Bousfield's $K$-localization functor $L_{K}$ [Bou] to identify $J K(\mathbb{Z})$ with $L_{K}\left(B G L(\mathbb{Z})^{+}\right)$.

Where not otherwise stated, all spaces are supposed localized at 2 in what follows.

\section{The 2-complete theory}

The natural procedure is in any case to begin with the 2-completed theory, and proceed to integrate it with the rational to obtain a 2-local statement. With this in mind, we begin with the following commutative diagram:

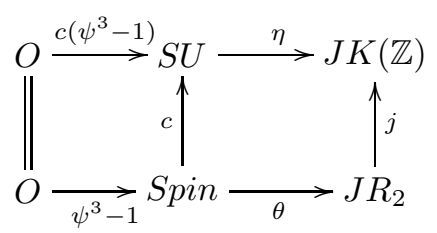

Both rows in this diagram are fibrations; the top row derived in the obvious way from the fibration (1), the second similarly from the definition of $J R_{2}$. The two squares are commutative by construction (compare the diagram on p.8 of [R2]); and the right hand square is fibred. The most 'natural' approach for such $K$-theory computations is usually via the geometric spectral sequence of Rothenberg-Steenrod (see e.g. $[\mathrm{A}-\mathrm{H}]$ ), which gives the $K$-theory of a quotient of groups (for example) in terms of those of the group and subgroup; and we can apply this spectral sequence to the fibre square provided that one of the Spin-actions is free. It will 
be convenient to suppose this for the action on $S U$ (of course nearly free, but not quite...), by the usual device of replacing $S U$ by $S U \times E S p i n$. We are accordingly using a homotopy equivalence of $J K(\mathbb{Z})$ with $(S U \times E S p i n) \times$ Spin $J R_{2}$; and we need the cohomology version of the Rothenberg-Steenrod sequence, which uses derived functors in the category of comodules over a coalgebra - in our case the coalgebra $K^{*}\left(\operatorname{Spin} ; \mathbb{Z}_{2}^{\hat{2}}\right)$.

Much of the argument can be simplified in this case, as we shall see, since we are dealing with a trivial comodule. To clarify the details of the application we want, i.e. to the 2 -complete $K$-cohomology, one or two technical points should be made. First, there is (as usual in $K$-cohomology of large spaces) the question of topology on the coalgebra and comodules $K^{*}\left(; \mathbb{Z}_{2}\right)$. Second, the coefficients are not a field, and the modules may not be free or even projective. We need to deal with these objections together so as to obtain a reasonable cotensor product functor. To begin with, the category $\mathcal{C}_{2}$ of profinite modules over $\mathbb{Z}_{2}$ is abelian, and the appropriate tensor product is the completed one, ' $\hat{\otimes}$ '. Because lim is exact in the category, the functor $-\hat{\otimes} A$ is exact if $A$ is an inverse limit of finitely generated free $\mathbb{Z}_{\hat{2}}$-modules. We shall call such a module 'flat', by analogy with the usual case. In particular, this applies to the Hopf algebras $K^{*}\left(\operatorname{Spin} ; \mathbb{Z}_{2} \hat{)}\right)$ and $K^{*}\left(S U ; \mathbb{Z}_{2}\right)$. (This is a consequence of $[\mathrm{H}]$, but the detail will be given later.) Hence $K^{*}\left(\quad ; \mathbb{Z}_{\hat{2}}\right)$ translates products of spaces into completed tensor products of $\mathbb{Z}_{2}$-algebras, when one of the spaces is $S U$ or Spin.

If $A$ is a (profinite) flat cocommutative $\mathbb{Z}_{2}$-coalgebra, and $B, C$ are compact comodules over $A$, we define the completed cotensor product $B \hat{\square}_{A} C$ to make the sequence (cf $[\mathrm{M}-\mathrm{M}]$ )

$$
0 \longrightarrow B \hat{\emptyset_{A}} C \longrightarrow B \hat{\otimes} C \stackrel{\Delta \otimes 1-1 \otimes \Delta}{\longrightarrow} B \hat{\otimes} A \hat{\otimes} C
$$

exact. ( $\Delta$ denotes the structural morphisms for the comodules.) This bifunctor is left exact on

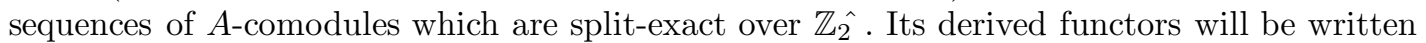
$\widehat{\operatorname{Cotor}}_{p}^{A}(B, C)$. Recall the spectral sequence - stated here in the appropriate form for our purpose.

Proposition 2.1. Let $G$ be a group, and let $X, Y$ be $G$-spaces with either $X$ or $Y$ free (all in a suitably small category, e.g. 2-local $C W$-complexes). If $G, X$ have $K^{*}\left(\quad ; \mathbb{Z}_{2}\right)$ flat in $\mathcal{C}_{2}$, then there is a strongly convergent spectral sequence with

$$
\begin{gathered}
E_{2}^{p}={\widehat{\operatorname{Cotor}_{K}^{*}\left(G ; \mathbb{Z}_{2}\right)}}^{p}\left(K^{*}\left(X ; \mathbb{Z}_{2} \hat{2}\right), K^{*}\left(Y ; \mathbb{Z}_{2} \hat{2}\right)\right) \\
E_{\infty} \sim K^{*}\left(X \times_{G} Y ; \mathbb{Z}_{2}\right)
\end{gathered}
$$

Its edge homomorphism is the 'standard' map

$$
\eta: K^{*}\left(X \times_{G} Y ; \mathbb{Z}_{2}\right) \rightarrow K^{*}\left(X ; \mathbb{Z}_{2}\right)_{K^{*}\left(G ; \mathbb{Z}_{2}\right)} K^{*}\left(Y ; \mathbb{Z}_{2}\right)
$$

(which follows from the definitions).

The proof is the usual geometric one, using the bar resolution. Again because the inverse limit is exact in $\mathcal{C}_{2}$ there are no convergence problems.

Note. Since we are interested in the 2-local theory, we shall also need a local version of this. Here arguments using profiniteness naturally break down, and alternative methods must be used. The best option is to use the corresponding sequence for $K$-homology, which involves the ordinary tensor product and the ordinary Tor groups over $K_{*}\left(G ; \mathbb{Z}_{2}\right)$. Again (since homology theories behave well with respect to direct limits) the sequence is strongly convergent; in this case the proof is clearly simpler. We then need to dualize the results in the appropriate way to derive the $K$-cohomology.

We are now ready to state the structure theorem for the map $c:$ Spin $\rightarrow S U$; for maximum generality we shall need the local version.

Proposition 2.2. (i) The Hopf algebras $K^{*}\left(\operatorname{Spin} ; \mathbb{Z}_{2}\right)$ resp. $K^{*}\left(S U ; \mathbb{Z}_{2}\right)$ are completed exterior algebras on submodules of primitive generators, say $P_{R}, P_{C}$ respectively; and the map 
c induces an epimorphism from $P_{C}$ to $P_{R}$, whose kernel $Q$ is a direct summand. Accordingly, writing $\hat{E}($ ) for the completed exterior algebra on primitive elements, we have:

$$
\begin{gathered}
K^{*}\left(\operatorname{Spin} ; \mathbb{Z}_{2}\right)=\hat{E}\left(P_{R}\right) \\
K^{*}\left(S U ; \mathbb{Z}_{2}\right)=\hat{E}\left(P_{C}\right) \cong \hat{E}\left(P_{R} \oplus Q\right)=\hat{E}\left(P_{R}\right) \hat{\otimes} E(Q)
\end{gathered}
$$

as a tensor product of Hopf algebras.

(ii) The same statements hold for $K$-theory with $\mathbb{Z}_{2}^{\hat{2}}$ coefficients, and $K^{*}\left(\operatorname{Spin} ; \mathbb{Z}_{2}^{\hat{2}}\right)$ resp. $K^{*}\left(S U ; \mathbb{Z}_{2}^{\hat{n}}\right)$ is isomorphic to $K^{*}\left(\operatorname{Spin} ; \mathbb{Z}_{2}\right) \otimes_{\mathbb{Z}_{2}} \mathbb{Z}_{2}$ resp. $K^{*}\left(S U ; \mathbb{Z}_{2}\right) \otimes_{\mathbb{Z}_{2}} \mathbb{Z}_{2}$

From this will follow:

Proposition 2.3. For any space $X$ with an action of Spin, the edge homomorphism of the spectral sequence defines a natural isomorphism

$$
\eta: K^{*}\left((S U \times E \text { Spin }) \underset{\text { Spin }}{\times} X ; \mathbb{Z}_{2}\right) \rightarrow \hat{E}(Q) \hat{\otimes} K^{*}\left(X ; \mathbb{Z}_{\hat{2}}\right)
$$

We postpone the proof of proposition 2.2 to the next section, and show that it implies proposition 2.3 .

For this, it is sufficient to identify $K^{*}\left(S U ; \mathbb{Z}_{2}\right) \hat{\square}_{K^{*}\left(S p i n ; \mathbb{Z}_{\hat{2}}\right)} K^{*}\left(X ; \mathbb{Z}_{2}\right)$. From the splitting of proposition 2.2 , we can deduce that

$$
\mu^{*}: K^{*}\left(S U ; \mathbb{Z}_{2}\right) \rightarrow K^{*}\left(\operatorname{Spin} ; \mathbb{Z}_{2}\right) \hat{\otimes} K^{*}\left(S U ; \mathbb{Z}_{2}\right)
$$

is identified with

$$
\hat{E}\left(P_{R}\right) \hat{\otimes} E(Q) \stackrel{\Delta \otimes 1}{\longrightarrow} \hat{E}\left(P_{R}\right) \hat{\otimes} \hat{E}\left(P_{R}\right) \hat{\otimes} \hat{E}(Q)
$$

Now we know that $\left(\hat{E}\left(P_{R}\right) \hat{\otimes} \hat{E}(Q)\right) \hat{\square}_{\hat{E}\left(P_{R}\right)} K^{*}\left(X ; \mathbb{Z}_{2}\right) \cong \hat{E}(Q) \hat{\otimes} K^{*}\left(X ; \mathbb{Z}_{2}\right)$; in fact, this is the dual of the well-known analogous formula for the tensor product, and the isomorphism is natural. However, $\hat{E}(Q) \hat{\otimes} K^{*}\left(X ; \mathbb{Z}_{2} \hat{)}\right)$ is exact as a functor of the comodule $K^{*}\left(X ; \mathbb{Z}_{2}^{\hat{2}}\right)$, since $\hat{E}(Q)$ is flat, and so its derived functors are trivial:

$$
\begin{gathered}
\widehat{\operatorname{Cotor}}_{K^{*}(\operatorname{Spin} ; \hat{\mathbb{2}})}^{0}\left(K^{*}\left(X ; \mathbb{Z}_{2} \hat{)}\right), K^{*}\left(S U ; \mathbb{Z}_{2} \hat{)}\right)=K^{*}\left(X ; \mathbb{Z}_{\hat{2}}\right) \hat{\otimes} \hat{E}(Q)\right. \\
\widehat{\operatorname{Cotor}}_{K^{*}\left(\operatorname{Spin} ; \mathbb{Z}_{\hat{2}}\right)}\left(K^{*}\left(X ; \mathbb{Z}_{\hat{2}}\right), K^{*}\left(S U ; \mathbb{Z}_{2}\right)\right)=0 \quad(p>0)
\end{gathered}
$$

Using the edge homomorphism of the spectral sequence, proposition 2.3 follows.

\section{Structure of $K^{*}(\operatorname{Spin}), K^{*}(S U)$}

We now proceed to the proof of proposition 2.2. Let $\lambda_{r}^{i}$ resp. $\lambda_{c}^{i}$ be the $i$ th 'stabilized' exterior power of the standard representation $\theta$ from $\operatorname{Spin}(2 n+1)$ resp. $S U(2 n+1)$ to $U$, considered as an element of the representation ring. That is, $\lambda_{r}^{i}$ is the result of applying the operation $\lambda^{i}$ to $\theta-(2 n+1)$. Then it is obvious that under inclusion maps of $\operatorname{Spin}(2 n+1)$ 's and $S U(2 n+1)$ 's the $\lambda^{i}$ 's are preserved; and that $c^{*}\left(\lambda_{c}^{i}\right)=c^{*}\left(\bar{\lambda}_{c}^{i}\right)=\lambda_{r}^{i}$.

Let now $\beta$ be the operation (see $[\mathrm{H}]$ ) which to any representation $\rho$ of $G$ assigns its class $\beta(\rho)$ in $K^{1}(G)=[G, U]$ considered as a map from $G$ to $U$. The basic theorem of $[\mathrm{H}]$ gives us that $K^{*}\left(S U(2 n+1) ; \mathbb{Z}_{2}\right)$ is the exterior algebra

$$
E_{\mathbb{Z}_{2}}\left(\beta\left(\lambda_{c}^{1}\right), \ldots, \beta\left(\lambda_{c}^{n}\right), \beta\left(\bar{\lambda}_{c}^{1}\right), \ldots, \beta\left(\bar{\lambda}_{c}^{n}\right)\right)
$$

since these can be seen to be equivalent to the basic representations modulo a little manipulation. (The generators are also, as usual, the primitives for the Hopf algebra structure.) The similar result is not quite true for $\operatorname{Spin}(2 n+1)$, as is well known, the picture being complicated by the Spin representation $\Delta_{n}$, of dimension $2^{n}$. We have:

$$
K^{*}\left(\operatorname{Spin}(2 n+1) ; \mathbb{Z}_{2}\right)=E_{\mathbb{Z}_{2}}\left(\beta\left(\lambda_{r}^{1}\right), \ldots, \beta\left(\lambda_{r}^{n-1}\right), \beta\left(\Delta_{n}\right)\right)
$$


However, there is a relation between $\lambda_{r}^{n}$ and $\Delta_{n}$, since $\left(\Delta_{n}\right)^{2}=\lambda_{r}^{n}+$ a sum of terms in $\lambda_{r}^{1}, \ldots, \lambda_{r}^{n-1}$. Writing $\Delta_{n}=2^{n}+\tilde{\Delta}_{n}$, and applying the usual relations for $\beta$, we have that $\beta\left(\Delta_{n}\right)^{2}=2^{n+1} \beta\left(\Delta_{n}\right)$. Hence, $\beta\left(\lambda_{r}^{n}\right)=2^{n+1} \beta\left(\Delta_{n}\right)\left(\bmod \beta\left(\lambda_{r}^{1}\right), \ldots, \beta\left(\lambda_{r}^{n-1}\right)\right)$.

Write $M_{n}$ for the $\mathbb{Z}_{2}$-module which generates the exterior algebra $K^{*}\left(\operatorname{Spin}(2 n+1) ; \mathbb{Z}_{2}\right)$ and $N_{n}$ for the submodule generated by the $\beta\left(\lambda_{r}^{i}\right)$ 's. We can deduce a short exact sequence

$$
0 \rightarrow N_{n} \rightarrow M_{n} \rightarrow \mathbb{Z} / 2^{n+1} \cdot \beta\left(\Delta_{n}\right) \rightarrow 0
$$

The restrictions from $E_{n+1}$ to $E_{n}$ are straightforward if we take into account that $\Delta_{n+1}$ restricts to $2 . \Delta_{n}$. Hence the map from $\mathbb{Z} / 2^{n+2}$ to $\mathbb{Z} / 2^{n+1}$ in the above sequence multiplies the generator by 2 . It is easy to deduce that the inverse limit of the $\mathbb{Z} / 2^{n+1}$ 's is zero; and so (since they are finite) is the $\lim _{\leftarrow}^{1}$. Hence the map from $\lim _{\leftarrow}\left\{N_{n}\right\}$ to $\lim _{\leftarrow}\left\{M_{n}\right\}$ - the primitives of $K^{*}(\operatorname{Spin})$ - is an isomorphism, and we have:

Proposition 3.1. The K-cohomology rings of Spin, SU are as follows:

$$
\begin{gathered}
K^{*}\left(\operatorname{Spin} ; \mathbb{Z}_{2}\right)=\hat{E}_{\mathbb{Z}_{2}}\left(\beta\left(\lambda_{r}^{1}\right), \beta\left(\lambda_{r}^{2}\right), \ldots\right) \\
K^{*}\left(S U ; \mathbb{Z}_{2}\right)=\hat{E}_{\mathbb{Z}_{2}}\left(\beta\left(\lambda_{c}^{1}\right), \beta\left(\lambda_{c}^{2}\right), \ldots ; \beta\left(\bar{\lambda}_{c}^{1}\right), \beta\left(\bar{\lambda}_{c}^{2}\right) \ldots\right)
\end{gathered}
$$

and the restriction $c^{*}$ from $S U$ to Spin maps $\beta\left(\lambda_{c}^{i}\right), \beta\left(\bar{\lambda}_{c}^{i}\right)$ to $\beta\left(\lambda_{r}^{i}\right)(i=1,2, \ldots)$

From this, proposition 2.2 clearly follows.

We next deduce:

Proposition 3.2. The local $K$-theory of the quotient is given by

$$
K^{*}\left((S U \times E \text { Spin }) / \operatorname{Spin} ; \mathbb{Z}_{2}\right) \cong \hat{E}_{\mathbb{Z}_{2}}\left(\beta\left(\lambda_{c}^{1}\right)-\beta\left(\bar{\lambda}_{c}^{1}\right), \ldots\right) \cong \hat{E}_{\mathbb{Z}_{2}}(Q)
$$

in the terminology of proposition 2.2.

Proof. As stated above, the best way to prove this is as follows. First, dualize proposition 3.1 to give a result on the local $K$-homology (the map $c$ now induces a split monomorphism). Next, apply the Rothenberg-Steenrod sequence in local $K$-homology; this is well-behaved, and strong convergence is easily established, as well as flatness (in the usual sense) for the $K$ algebras involved. We find a natural isomorphism in $K$-homology in a form dual to that of proposition 2.3. In the special case where $X$ is a point, this can now simply be dualized back to give the required result.

This procedure is of course roundabout, but seems preferable to developing a theory if topological modules which will deal properly with very large algebras over $\mathbb{Z}_{2}$ of the kind we are considering here in $K$-cohomology.

\section{The $K$-theory of $J K(\mathbb{Z})$}

We are now in a position to put the pieces together. The key point is that $J R_{2}$ is a 2 -adic space, so the local theory and the 2-adic theory coincide for it.

Theorem 4.1. There is a natural isomorphism:

$$
\begin{aligned}
K^{*}\left(J K(\mathbb{Z}) ; \mathbb{Z}_{2}\right) & \cong \hat{E}_{\mathbb{Z}_{2}}(Q) \hat{\otimes} K^{*}\left(J R_{2} ; \mathbb{Z}_{2}\right) \\
& \cong K^{*}\left(B B S O ; \mathbb{Z}_{2}\right) \hat{\otimes} K^{*}\left(J R_{2} ; \mathbb{Z}_{2}\right)
\end{aligned}
$$

with an analogous isomorphism for $\mathbb{Z}_{\hat{2}}$ coefficients.

Proof. We'd like to use a basepoint in $J R_{2}$, but of course can't suppose there is one which is fixed under Spin. Consider instead the equivariant embedding of $J R_{2}$ in the unreduced cone $C^{+} J R_{2}$. If we can prove the result for $C^{+} J R_{2}$ and for the pair $P=\left(C^{+} J R_{2}, J R_{2}\right)$ 
separately, then it will follow for $J R_{2}$ by the 5-lemma. Now for $C^{+} J R_{2}$ it is already proved (by proposition 3.2 ). For $P$, we consider the commutative diagram:

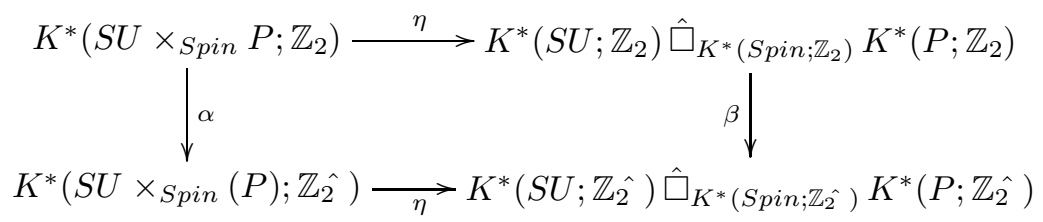

The arrow $\eta$ in the lower row is an isomorphism by proposition 2.3. The two vertical arrows are induced by the coefficient homomorphism. Since the reduced homology of $J R_{2}$ is finite in every dimension, the same is true for the pair $S U \times_{S p i n} P$; so $K^{*}\left(S U \times_{S p i n} P ; \mathbb{Z}_{2}\right)$ is a 2 -adic group. Hence the arrow marked $\alpha$ is an isomorphism. On the other hand, we can embed $K^{*}\left(S U ; \mathbb{Z}_{2}\right) \hat{\square}_{K^{*}\left(\operatorname{Spin} ; \mathbb{Z}_{2}\right)} K^{*}\left(P ; \mathbb{Z}_{2}\right)$ in $K^{*}\left(S U ; \mathbb{Z}_{2}\right) \hat{\otimes} K^{*}\left(P ; \mathbb{Z}_{2}\right)$ and identify the latter with

$$
K^{*}\left(S U ; \mathbb{Z}_{2}\right) \hat{\otimes}\left(\mathbb{Z}_{2} \hat{\otimes} K^{*}\left(P ; \mathbb{Z}_{2}\right)\right)
$$

(again because $K^{*}\left(P ; \mathbb{Z}_{2}\right)$ is 2-adic). Using this, and the definition of the cotensor product, we find that the right hand vertical arrow $\beta$ is also an isomorphism. Hence the upper arrow $\eta$ is one.

Now by the argument used in proposition 2.3 , this implies that $K^{*}\left(S U \times \times_{S p i n} P ; \mathbb{Z}_{2}\right)$ is isomorphic to $\hat{E}_{\mathbb{Z}_{2}}(Q) \hat{\otimes} K^{*}\left(P ; \mathbb{Z}_{2}\right)$. This proves the first line of the theorem. The second results from

Lemma 4.1. The fibre SU/Spin of $c: B S p i n \rightarrow B S U$ can be identified with the Hopf map $\eta: B B S O \rightarrow B$ Spin.

Proof. The composite $c \circ \eta$ is trivial and so lifts to a map $\tilde{\eta}: B B S O \rightarrow F i b(c)$. A check on the homotopy sequence shows that this is a homotopy equivalence.

A comparison with the fibre sequence (2) shows that the sequence splits from the viewpoint of 2-local $K$-theory. Finally, it is worth noting that the $K$-theory of $J R_{2}$ has been known for a long time, see $[\mathrm{H}-\mathrm{S}]$; it is essentially the completed representation ring of the infinite symmetric group $\Sigma_{\infty}$.

\section{The results for $B G L(\mathbb{Z})^{+}$}

As was remarked in $\S 1$, theorem 4.1 immediately gives us the 2 -adic $K$-theory of $B G L(\mathbb{Z})^{+}$, since $\left(B G L(\mathbb{Z})^{+}\right)_{2}$ is homotopy equivalent to $J K(\mathbb{Z})_{2}$. To deal with the localizations, we shall prove the following result:

Theorem 5.1. Let $L_{K}$ denote Bousfield's $K$-theory localization functor on spaces [Bou]. There is a homotopy equivalence of $J K(\mathbb{Z})$ with $L_{K}\left(B G L(\mathbb{Z})^{+}\right)$.

Since $K^{*}\left(L_{K}\left(X ; \mathbb{Z}_{2}\right)\right) \cong K^{*}\left(X ; \mathbb{Z}_{2}\right)$ for any $X$, this shows:

Corollary 5.1. The $K$-theory of $B G L(\mathbb{Z})^{+}$with $\mathbb{Z}_{2}$ or $\mathbb{Z}_{2}$ coefficients is computed by theorem 4.1.

For the proof of theorem 5.1, we shall simplify notation by writing $K(\mathbb{Z})$ for the ring $\mathbb{Z} \times B G L(\mathbb{Z})^{+}$. We follow the arguments of [Bö] and ( $\$ 2$ of) [R1]. In [Bö], the rational component is considered as well as the 2-adic, but the discussion is essentially concerned with $\Omega K(\mathbb{Z})$; while in [R1], the argument is at the level of spectra, but is purely 2-adic. Our concern is to use $K$-localization to circumvent these restrictions.

We first define a map $s$ as the composite $B S O \stackrel{i}{\longrightarrow} B S G \stackrel{\eta}{\longrightarrow} S G$, where $i$ is the 'forgetful' map from bundles to spherical fibrations, and $\eta$ is multiplication by the Hopf map. Both maps 
are 2-locally defined, and are infinite loop maps. If $r: S G \rightarrow K(\mathbb{Z})_{1}$ is the map into the 'one-component' induced by $Q S^{0} \rightarrow K(\mathbb{Z})$, then $r$ is again a local infinite loop map. In [R1] it is shown that the 2-completion of $r \circ s$ is nullhomotopic at the level of spectra. However, since $S G$ has finite homotopy groups, $r \circ s$ is 2-locally trivial if it is trivial after completion; and as in [R1], the nullhomotopy deloops to give a map $g: B f i b(s) \rightarrow K(\mathbb{Z})_{1}$.

Lemma 5.1. The localizations $L_{K}(B f i b(s))$ and $L_{K}(J K(\mathbb{Z}))$ are homotopy equivalent.

Proof. This follows directly from diagram 2.8 of [R1], since the fibre sequence $C_{\otimes} \rightarrow$ $B f i b(s) \rightarrow J K(\mathbb{Z})$ can be constructed 2-locally using $\rho^{3}$. Since $L_{K}\left(C_{\otimes}\right)$ is a point by [H-S], the fibration becomes an equivalence after $K$-localization.

Finally, we need:

Lemma 5.2. The $K$-theory localization of $g, L_{K}(g)$, is a homotopy equivalence from $L_{K}(B f i b(s))$ to $L_{K}\left(K(\mathbb{Z})_{1}\right)$.

Proof. Since the homotopy groups in each case are finitely generated, it will be enough to show that $g$ induces isomorphisms on homotopy (a) when 2-completed and (b) when tensored with $\mathbb{Q}$. For the 2-completion, we again use Rognes' diagram 2.8. The preceding lemma implies that the map there called ' $h$ ' exists after $K$-localization; we shall call it ' $L_{K}(h)$ ', ignoring the question of whether $h$ exists. And $L_{K}(g)$ is an equivalence if and only if $L_{K}(h)$ is. But by the subsequent arguments of Rognes, $L_{K}(h)$ is a right inverse for the more usual map $\Phi: L_{K}\left(K(\mathbb{Z})_{1}\right)_{2} \rightarrow L_{K} J K(\mathbb{Z})_{2}$. (As pointed out in [R1], this involves an essentially 2-adic argument.) We now know, however, that $\Phi$ is a 2 -adic equivalence, and so $L_{K}(h)$ is. For the rational version we use the argument on pp.31-2 of [Bö]; the homotopy groups of $f i b(s)_{2}$ and $\left(\Omega K(\mathbb{Z})_{1}\right)_{2}$ are the same after inverting 2 , and hence $\Omega g$ is an isomorphism on rational homotopy. The same result therefore follows for $g$ and so for $L_{K}(g)$.

\section{References}

[A-H] Anderson, D.M. and Hodgkin, L.H., On the K-theory of Eilenberg-MacLane complexes, Topology 7 (1968), 317-329.

[A-M-N-Y] Arlettaz, D., Mimura, M., Nakahata, K. and Yagita, K. , The mod 2 cohomology of the linear groups over the ring of integers Proc. Amer. Math. Soc. 127 (1999), 2199-2212.

[B-L] Bloch, S. and Lichtenbaum, S. , A spectral sequence for motivic cohomology, $K$-theory preprint archive no. 62 (1994).

[Bö] Bökstedt, M, The rational homotopy type of $\Omega W h^{\text {Diff }}(*)$, in Algebraic Topology, Aarhus 1982 (Lect. Notes Math. vol. 1051), Berlin Heidelberg New York, Springer, 1984, 25-37.

[B-M] Bökstedt, M. and Madsen, I., Topological cyclic homology of the integers Astérisque 226 (1994), 57-143.

[Bou] Bousfield, A.K., The localization of spectra with respect to homology Topology 18 (1979), 257-281.

[D-F] Dwyer, W. and Friedlander, E. , Conjectural calculations of general linear group cohomology, Applications of Algebraic $K$-theory to Algebraic Geometry and Number Theory, Boulder 1983 (Contemp. Math. vol. 55 part I), AMS Providence, 1986, 135-147.

[D-M] Dwyer, W. and Mitchell, S.A., On the K-theory spectrum of a ring of algebraic integers K-theory 14 (1998), 201-263.

[H] Hodgkin, L.H., K-theory of Lie groups Topology 6 (1967), 1-36 . 
[H-S] Hodgkin, L.H. and Snaith, V.P., The K-theory of some more well-known spaces Illinois J. Math. 22 (1978), 270-278 .

[L] Lichtenbaum, S., Values of zeta functions, étale cohomology, and algebraic $K$ theory, Classical Algebraic $K$-theory and Connections with Arithmetic. (Lect. Notes in Math. vol. 342), Berlin Heidelberg New York, Springer, 1973, 489-501.

[Ma] May, J.P. $E_{\infty}$ Ring Spaces and $E_{\infty}$ Ring Spectra (Lect. Notes in Math. vol. 577), Berlin Heidelberg New York, Springer, 1977.

[M-M] Milnor, J.W. and Moore, J.C., On the Structure of Hopf algebras Ann. of Math. 81 (1965), 211-264 .

[Mi1] Mitchell, S.A., Topological K-theory of algebraic $K$-theory spectra, $K$-theory preprint archive no.345 (1999) .

[Mi2] Mitchell, S.A., K-theory hypercohomology spectra of number rings at the prime 2, $K$-theory preprint archive no. 386 (1999) .

[Ø] Østvær, P.-A., On algebraic K-theory spectra, preprint .

[Q] Quillen, D., On the cohomology and K-theory of the general linear groups over a finite field Ann. of Math. 96 (1972), 552-586 .

[R1] Rognes, J. , The Hatcher-Waldhausen map is a spectrum map Math. Ann. 299 (1994), 529-549 .

[R2] Rognes, J., Algebraic K-theory of the two-adic integers J. Pure Applied Algebra 134 (1999), 287-326 .

[R-W-K] Rognes, J., Weibel, C., and Kolster, M. , 2-primary algebraic K-theory of rings of integers in number fields J. Amer. Math. Soc. 13 (2000), 1-54 .

[R-W] Rognes, J. and Weibel, C. , Étale descent for 2-primary algebraic K-theory of totally imaginary number fields K-theory 16 (1999), 101-104.

[S] Sullivan, D., Genetics of homotopy theory and the Adams conjecture Ann. of Math. 100 (1974), 1-79 .

[S-V] Suslin, A. and Voevodsky, V. , Bloch-Kato conjecture and motivic cohomology with finite coefficients, $K$-theory preprint archive no. 83 (1995); see also Friedlander, E., Motivic complexes of Suslin and Voevodsky, Astérisque 245 (1997), $355-378$.

[V] Voevodsky, V. , The Milnor conjecture, K-theory preprint archive no. 170 (1996); see also Kahn, B., The Milnor conjecture after V. Voevodsky, Astérisque 245 (1997), 379-418 .

[W] Weibel, C. , The 2-torsion in the $K$-theory of the integers, $K$-theory preprint archive no.141 (1996).

This article may be accessed via WWW at http://www.rmi.acnet.ge/hha/ or by anonymous ftp at ftp://ftp.rmi.acnet.ge/pub/hha/volumes/2000/n9/n9.(dvi,ps,dvi.gz,ps.gz)

Luke Hodgkin luke.hodgkin@kcl.ac.uk

King's College

London 